\title{
Dynamic Error Analysis Method for Vibration Shape Reconstruction of Smart FBG Plate Structure
}

\author{
Hesheng Zhang, ${ }^{1,2}$ Xiaojin Zhu, ${ }^{1}$ Zhiyuan Gao, ${ }^{1}$ Kaining Liu, ${ }^{1}$ and Zhaowen Li ${ }^{1}$ \\ ${ }^{1}$ School of Mechatronic Engineering and Automation, Shanghai University, Shanghai 200072, China \\ ${ }^{2}$ The 804th Institute, Shanghai Academy of Space Flight Technology, CASC, Shanghai 201109, China
}

Correspondence should be addressed to Xiaojin Zhu; mgzhuxj@shu.edu.cn

Received 26 July 2015; Revised 6 October 2015; Accepted 18 October 2015

Academic Editor: Peng Chen

Copyright (C) 2016 Hesheng Zhang et al. This is an open access article distributed under the Creative Commons Attribution License, which permits unrestricted use, distribution, and reproduction in any medium, provided the original work is properly cited.

\begin{abstract}
Shape reconstruction of aerospace plate structure is an important issue for safe operation of aerospace vehicles. One way to achieve such reconstruction is by constructing smart fiber Bragg grating (FBG) plate structure with discrete distributed FBG sensor arrays using reconstruction algorithms in which error analysis of reconstruction algorithm is a key link. Considering that traditional error analysis methods can only deal with static data, a new dynamic data error analysis method are proposed based on LMS algorithm for shape reconstruction of smart FBG plate structure. Firstly, smart FBG structure and orthogonal curved network based reconstruction method is introduced. Then, a dynamic error analysis model is proposed for dynamic reconstruction error analysis. Thirdly, the parameter identification is done for the proposed dynamic error analysis model based on least mean square (LMS) algorithm. Finally, an experimental verification platform is constructed and experimental dynamic reconstruction analysis is done. Experimental results show that the dynamic characteristics of the reconstruction performance for plate structure can be obtained accurately based on the proposed dynamic error analysis method. The proposed method can also be used for other data acquisition systems and data processing systems as a general error analysis method.
\end{abstract}

\section{Introduction}

Plate structure is a common structure form of aircrafts and aerospace vehicles [1-4]. Shape reconstruction of such structure can be achieved by nonvisual methods using distributed FBG sensors, which generally uses a FBG sensor network or a FBG sensor array [5-7]. Compared with visual perception method [8-11], nonvisual perception method needs less amount of collection data, with high acquisition accuracy, high real-time performance, and high anti-interference capability. Currently there are two types of methods: modal method [5, 12-17] and geometrical method [18-22].

Displacement mode superposition method mentioned in $[5,17,23,24]$ is only suitable for shape reconstruction with small amplitude distortion as the strain direction on the measuring surface is uncertain. Geometrical iteration method mentioned in $[19,25,26]$ uses curvature data along a single direction and plane curve fitting algorithm to achieve shape reconstruction. Although these methods have good reconstruction performance for pure bending deformation, they are not suited for torsional deformation. The authors of [26] have achieved curved surface reconstruction, but the method is not suited for real-time reconstruction as it needs to solve complex nonlinear equations. The authors of [19] proposed a space curve shape reconstruction algorithm, but it is not suited for bend torsion coupling shape reconstruction. To overcome the shortcomings of the above algorithms, orthogonal curve net based space surface reconstruction algorithm can be employed by using biorthogonal strain data and orthogonal curve net to achieve shape reconstruction of complex vibration shape. Recursive computation can be employed for real-time implementation instead of solving nonlinear equations.

While performance comparison is mentioned in $[5,12-$ $22,24]$, static error analysis such as mean square error between real deformation and theoretically calculated deformation is used $[19,23]$. However, there is no dynamic performance comparison method to value these proposed methods to provide transmission performance. While structure vibration reconstruction can be understood as a transformation 
process of the original strain data, it can be treated as a kind of generalized signal transmission. So dynamic data transmission features can be defined including signal amplitude attenuation, phase delay, and noise mixing [27, 28]. So accurate dynamic data error analysis can be done using system identification methods by taking the vibration response as the input of the reconstruction system and the reconstruction results as the output. Least mean square (LMS) is a famous algorithm for adaptive control [29-31], and many applications for system identification can be found in [32-34].

Taking a one end fixed square flexible plate as experimental plate, a new dynamic data error analysis method is proposed based on LMS algorithm. The proposed method can also be used for other data acquisition systems and data processing systems as a general error analysis method. Section 2 introduces the orthogonal curved network based reconstruction method. Section 3 introduces the construction of the experimental smart FBG plate and the fundamental principles of FBG sensing. Section 4 proposes a dynamic error analysis model for dynamic reconstruction error analysis and gives the detailed modelling method including signal amplitude attenuation, phase delay, and noise mixing. Section 5 illustrates the parameter identification for the proposed dynamic error analysis model and how to design filter $F$. Section 6 introduces the simulation and experimental platform. Section 7 is the simulation and experiment results. Section 8 comes to the conclusion.

\section{Orthogonal Curved Network Based Reconstruction Method}

The basic process of curved surface reconstruction algorithm based on orthogonal curved network is as follows: constructing the orthogonal curved network by using continuous curvature and establishing the orthogonal moving coordinate system on two directions for orthogonal curved network; calculating node coordinates of orthogonal curvilinear net using the moving coordinate system and using the internal relations of different nodes to implement the coupling relationship between orthogonal coordinate systems. The iterative calculation of node coordinates and the transformation of moving coordinate system to realize all nodes' coordinate of orthogonal curved network constitute the two steps used to reconstruct the whole shape of curved surface.

2.1. Establishing Orthogonal Curved Network and Moving Coordinate System. According to differential geometry, a regular parametric curved surface $S$ is a continuous mapping from an area $D$ on space $E^{2}$ to space $E^{3}$. Cartesian coordinate system can be established on $E^{2}$ and $E^{3}$ separately. Using $(u, v)$ to denote the coordinate in $E^{2}$ and using $(x, y, z)$ to denote the coordinate in $E^{3}$,

$$
S=f(x(u, v), y(u, v), z(u, v)) .
$$

On the basis of curvature continuity, those measuring points which have equal space (denoted by $\Delta s$ ) between each other were chosen. The measuring points were connecting

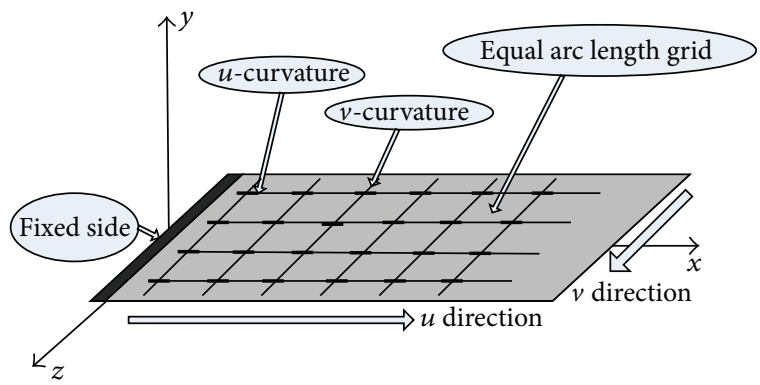

FIGURE 1: Schematic diagram of partial equal arc length grid.

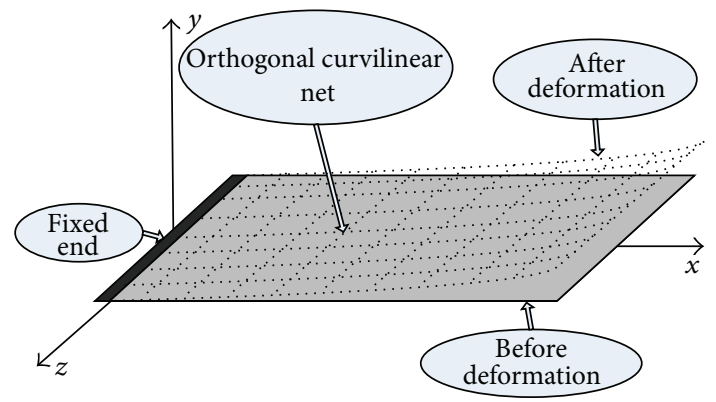

FIGURE 2: Orthogonal curve net.

from $u$ direction and $v$ direction separately. Then the orthogonal equal arc length grid is shown in Figure 1.

The orthogonal curvature of measuring points is divided into the curvature along $u$ direction and the curvature along $v$ direction, which are denoted by $\rho_{u}(n, m)$ and $\rho_{v}(n, m)$ separately, where $(n, m)$ is the point ordinal. For convenient description, $p(n, m)$ is used to denote discrete points on curved surface, where $n$ is the ordinal along $u$ direction and $m$ is the ordinal along $v$ direction.

A length-preserving correspondence can be established between deformed curved surface and original curved surface because there is a one-to-one correspondence of every point between pre- and postdeformation, and the orthogonal relationship between two curves through nodes will not change under a micro deformation condition. So, the equal arc length grids mentioned above became an orthogonal curve net as shown in Figure 2.

If the coordinate of each node on orthogonal curve net shown in Figure 2 can be solved separately by a certain computation method, the whole smooth curved surface can be obtained by traditional curved surface fitting algorithm. Considering that the research object is one-side fixed and there is no large deformation, there exist two boundary conditions:

(1) There is no deformation on the fixed side of curved surface.

(2) The central line along $u$ direction of orthogonal curve net is plane curve.

In order to describe the algorithm conveniently, the moving coordinate system is established along the $u$ direction 


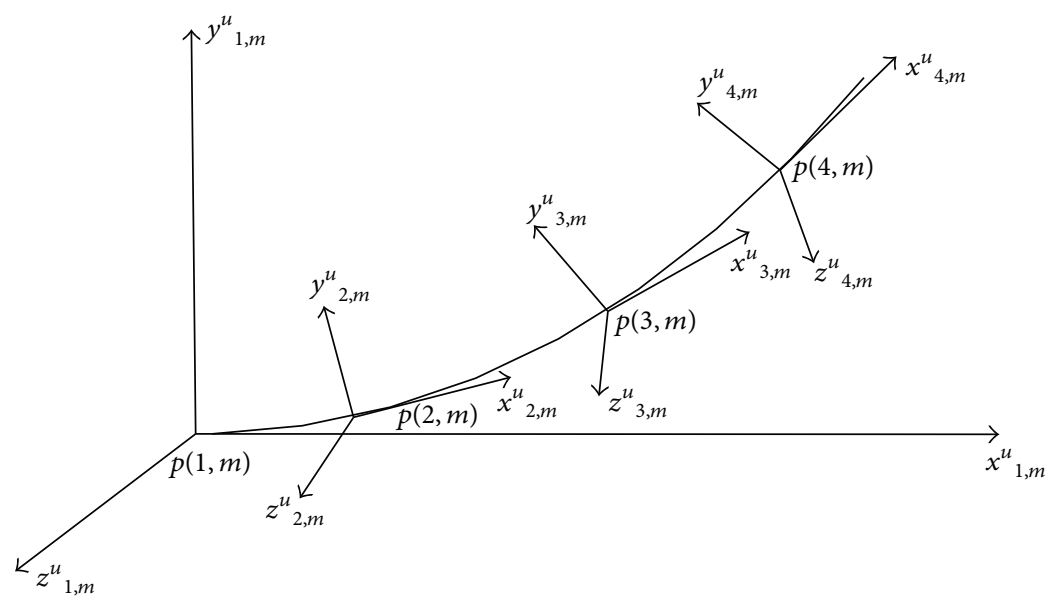

FIgURE 3: Moving coordinate system.

and $v$ direction of orthogonal curve net separately as shown in Figure 3.

To ensure the curve continuity, there is a coordinate transformation relationship between adjacent moving coordinate system on the curve. The relationship contains translation, rotation around $z$-axis, and rotation around $x$-axis, as shown in Figure 4.

In Figure $4, \theta_{u}(n, m)=\rho_{u}(n, m) \cdot \Delta s$, where $\varphi_{u}(n, m)$ is torsion angle. The transformation process is moving the coordinate origin from point $p(n, m)$ to point $p(n+1, m)$ firstly, then rotating $\theta_{u}(n, m)$ degrees around $z$-axis, and finally rotating $\varphi(n, m)$ degrees around $x$-axis. The torsion angle $\varphi_{u}(n, m)$ is obtained by the curvature coupling relationship between two orthogonal directions.

2.2. Nodal Coordinate Computation. Nodal coordinate recursive computation is the key part of the space curved surface reconstruction algorithm. Because the central line along $u$ direction is plane curve which can be obtained precisely by plate curved fitting algorithm, on the condition of using the central line as the boundary, nodal coordinate of the other curve can be obtained by iterative calculation. The micro arc between point $p(n, m)$ and point $p(n+1, m)$ is circle arc. $p(n, m) \cdot x, p(n, m) \cdot y$ and $p(n, m) \cdot z$ is used to denote the coordinates of point $p(n, m)$ separately. It is easy to know that, in the moving coordinate system that includes point $p(n, m)$, the coordinate $p^{\prime}(n+1, m)$ of point $p(n+1, m)$ is

$$
\begin{aligned}
& p^{\prime}(n+1, m) \cdot x=\frac{\sin \left(\theta_{u}(n, m)\right)}{\rho_{u}(n, m)}, \\
& p^{\prime}(n+1, m) \cdot y=\frac{1-\cos \left(\theta_{u}(n, m)\right)}{\rho_{u}(n, m)}, \\
& p^{\prime}(n+1, m) \cdot z=0 .
\end{aligned}
$$

Here, $p(n+1, m)$ is the coordinate of point $n$ in absolute coordinate system and $p^{\prime}(n+1, m)$ is the coordinate of point $n$ in the moving coordinate system. Let point $p(1, m)$ be the first point of the curve. The global coordinate system (absolute

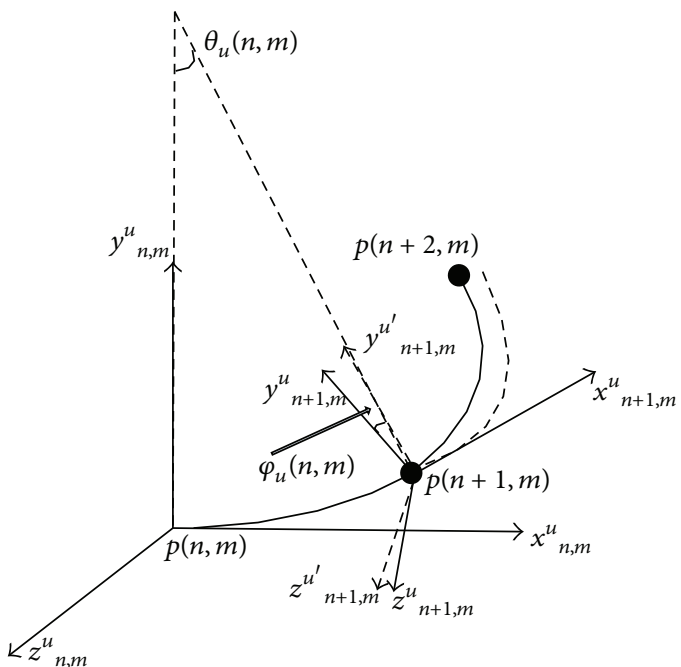

FIGURE 4: Moving coordinate system transformation.

coordinate system) is established by using point $p(1, m)$ as the original point. So, the absolute coordinate of point $p(2, m)$ can be obtained by formula (2):

$$
\begin{aligned}
& p(2, m) \cdot x=\frac{\sin \left(\theta_{u}(1, m)\right)}{\rho_{u}(1, m)}, \\
& p(2, m) \cdot y=\frac{1-\cos \left(\theta_{u}(1, m)\right)}{\rho_{u}(1, m)}, \\
& p(2, m) \cdot z=0 .
\end{aligned}
$$

Because the moving coordinate system whose original point is point $p(2, m)$ has been transformed,

(1) rotate $\theta_{u}(1, m)$ degrees around $z$-axis,

(2) rotate $\varphi_{u}(1, m)$ degrees around $x$-axis,

(3) move the original point from point $p(1, m)$ to point $p(2, m)$. 
So, if $A_{z}(\theta, m)$ denotes the rotation transformation matrix that shows that one point rotates $\theta$ degrees around $z$ axis, the coordinate of point $p^{\prime}(3, m)$ in absolute coordinate system is

$$
\begin{aligned}
p(3, m)= & A_{z}\left(\theta_{u}(1, m)\right) \cdot\left[A_{x}\left(\varphi_{u}(1, m)\right) \cdot p^{\prime}(3, m)\right] \\
& +p(2, m) .
\end{aligned}
$$

Similarly, the moving coordinate system where point $p(4, m)$ is located has been transformed relative to absolute coordinate system as the following steps:

(1) Rotate $\theta_{u}(1, m)$ degrees around $z$-axis.

(2) Rotate $\varphi_{u}(1, m)$ degrees around $x$-axis.

(3) Move the original point from point $p(1, m)$ to point $p(2, m)$.

(4) Continue to rotate $\theta_{u}(2, m)$ degrees around $z$-axis.

(5) Continue to rotate $\varphi_{u}(2, m)$ degrees around $x$-axis.

(6) Move the original point from point $p(2, m)$ to point $p(3, m)$.

From the above transformation, the absolute coordinate of $p(4, m)$ can be obtained:

$$
\begin{aligned}
& p(4, m)=A_{z}\left(\theta_{u}(1, m)\right) \cdot\left\{A_{x}\left(\varphi_{u}(1, m)\right)\right. \\
& \cdot\left\{A_{z}\left(\theta_{u}(2, m)\right) \cdot\left[A_{x}\left(\varphi_{u}(2, m)\right) \cdot p^{\prime}(4, m)\right]\right. \\
& \left.\left.+p^{\prime}(3, m)\right\}\right\}+p(2, m) .
\end{aligned}
$$

The absolute coordinate (when $n>2$ ) of point $p(n)$ can be obtained:

$$
\begin{aligned}
p(n, m)= & {\left[\prod_{i=1}^{n-2} A_{z}\left(\theta_{u}(i, m)\right) \cdot A_{x}\left(\varphi_{u}(i, m)\right)\right] } \\
& \cdot p^{\prime}(n, m)+p(n-1, m) .
\end{aligned}
$$

In formula (6), $A_{x}\left(\varphi_{u}(n, m)\right), A_{z}\left(\theta_{u}(n, m)\right)$ are

$$
\begin{aligned}
& A_{x}(\varphi(n, m)) \\
& \quad\left\{\begin{array}{ccc}
1 & 0 & 0 \\
0 & \cos \left(\varphi_{u}(n, m)\right) & -\sin \left(\varphi_{u}(n, m)\right) \\
0 & \sin \left(\varphi_{u}(n, m)\right) & \cos \left(\varphi_{u}(n, m)\right)
\end{array}\right\}, \\
& A_{z}(\theta(n, m)) \\
& \quad=\left\{\begin{array}{ccc}
\cos \left(\theta_{u}(n, m)\right) & -\sin \left(\theta_{u}(n, m)\right) & 0 \\
\sin \left(\theta_{u}(n, m)\right) & \cos \left(\theta_{u}(n, m)\right) & 0 \\
0 & 0 & 1
\end{array}\right\} .
\end{aligned}
$$

One nodal coordinate can be computed according to formula (3) and formulas (6) (7). All nodal coordinates can be obtained by iterative recursion combined with coupling transformation of moving coordinate system.

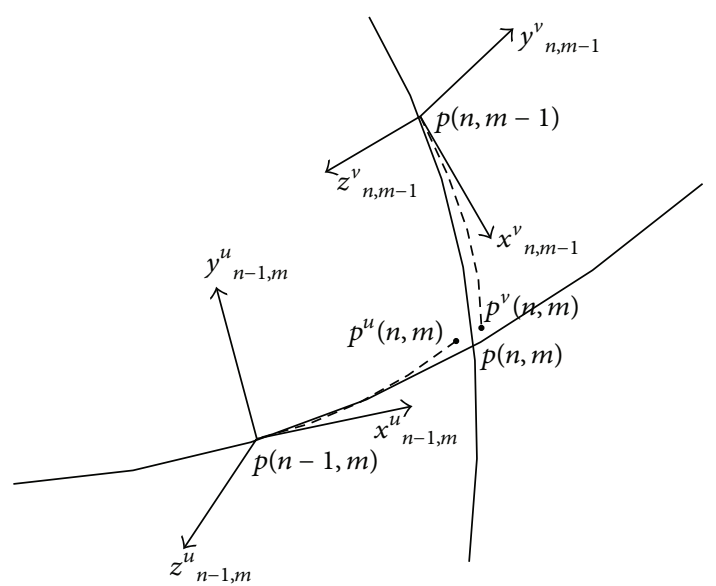

FIGURE 5: Coupling relationship between orthogonal coordinate directions.

2.3. Coupling Transformation of the Moving Coordinate Systems. The moving coordinate systems along $u$ direction and along $v$ direction are moving along the curve on which they are located. And they converge on the next node of orthogonal curve net. On the basis of a nodal coordinate used to compute the coordinate of next node, coupling transformation between two moving coordinate systems along two directions must be performed. If point $p^{u}(n, m)$ and $p^{v}(n, m)$ are the points in the moving coordinate system, the midpoint between points $p^{u}(n, m)$ and $p^{v}(n, m)$ is used as the valid value of node $p(n, m)$, as shown in Figure 5 . After the node $p(n, m)$ is obtained, the moving coordinate system along $u$ direction and $v$ direction converges on the node $p(n, m)$. Because two curves at node $p(n, m)$ are orthogonal, corresponding axes should converge. The torsion angle of two moving coordinate systems can be obtained.

Firstly, $x$-axis and $y$-axis of the two coordinate systems rotate $\theta_{u}(n-1, m)$ degrees and $\theta_{v}(n, m-1)$ degrees around their own $z$-axis separately. $\left(x_{n-1, m}^{u^{\prime}}, y_{n-1, m}^{u^{\prime}}, z_{n-1, m}^{u^{\prime}}\right)$ and $\left(x_{n, m-1}^{v^{\prime}}, y_{n, m-1}^{v^{\prime}}, z_{n, m-1}^{v^{\prime}}\right)$ are used to denote the obtained coordinate systems. The vector $x^{u^{\prime}}{ }_{n-1, m}$ denotes $x$-axis of the moving coordinate system along $u$ direction and so on. If $T\left(x_{n-1, m}^{u^{\prime}}, y_{n-1, m}^{u^{\prime}}, z_{n-1, m}^{u^{\prime}}\right) \cdot A$ is used to denote relative coordinate of vector $A$ in the coordinate system of $\left(x_{n-1, m}^{u^{\prime}}, y_{n-1, m}^{u^{\prime}}, z_{n-1, m}^{u^{\prime}}\right)$, the torsion angles of the two moving coordinate system can be obtained as

$$
\begin{aligned}
& \varphi_{u}(n-1, m) \\
& =\operatorname{arctg} \frac{\left[T\left(x_{n-1, m}^{u^{\prime}}, y_{n-1, m}^{u^{\prime}}, z_{n-1, m}^{u^{\prime}}\right) \cdot x_{n, m-1}^{v^{\prime}}\right] \cdot y}{\left|\left[T\left(x_{n-1, m}^{u^{\prime}}, y^{u^{\prime}}{ }_{n-1, m}, z^{u^{\prime}}{ }_{n-1, m}\right) \cdot x^{v^{\prime}}{ }_{n, m-1}\right] \cdot z\right|}, \\
& \varphi_{v}(n, m-1) \\
& =\operatorname{arctg} \frac{\left[T\left(x_{n, m-1}^{\nu^{\prime}}, y_{n, m-1}^{v^{\prime}}, z_{n, m-1}^{\nu^{\prime}}\right) \cdot z_{n-1, m}^{u^{\prime}}\right] \cdot y}{\left|\left[T\left(x_{n, m-1}^{\nu^{\prime}}, y_{n, m-1}^{v^{\prime}}, z_{n, m-1}^{\nu^{\prime}}\right) \cdot z_{n-1, m}^{u^{\prime}}\right] \cdot x\right|} .
\end{aligned}
$$


The two coordinate systems should rotate $\gamma$ degrees around their $y$-axis separately to ensure superposition of corresponding coordinate axis:

$$
\begin{aligned}
& \gamma_{u}(n-1, m)=-\gamma_{v}(n, m-1) \\
& =\frac{\arcsin \left(\left[T\left(x^{u^{\prime}}{ }_{n-1, m}, y^{u^{\prime}}{ }_{n-1, m}, z^{u^{\prime}}{ }_{n-1, m}\right) \cdot x^{v^{\prime}}{ }_{n, m-1}\right] \cdot x\right)}{2} .
\end{aligned}
$$

Thus, new moving coordinate system along $u$ direction can be obtained:

$$
\begin{aligned}
x_{n, m}^{u}= & A_{y}\left(\gamma_{u}(n-1, m)\right) \cdot A_{x}\left(\varphi_{u}(n-1, m)\right) \\
& \cdot A_{z}\left(\theta_{u}(n-1, m)\right) \cdot x_{n-1, m}^{u}, \\
y_{n, m}^{u}= & A_{y}\left(\gamma_{u}(n-1, m)\right) \cdot A_{x}\left(\varphi_{u}(n-1, m)\right) \\
& \cdot A_{z}\left(\theta_{u}(n-1, m)\right) \cdot y^{u}{ }_{n-1, m}, \\
z_{n, m}^{u}= & A_{y}\left(\gamma_{u}(n-1, m)\right) \cdot A_{x}\left(\varphi_{u}(n-1, m)\right) \\
& \cdot A_{z}\left(\theta_{u}(n-1, m)\right) \cdot z^{u}{ }_{n-1, m} .
\end{aligned}
$$

In the same way, new moving coordinate system along $v$ direction can also be obtained:

$$
\begin{aligned}
x_{n, m}^{v}= & A_{y}\left(\gamma_{v}(n, m-1)\right) \cdot A_{x}\left(\varphi_{v}(n, m-1)\right) \\
& \cdot A_{z}\left(\theta_{v}(n, m-1)\right) \cdot x_{n, m-1}^{v}, \\
y_{n, m}^{v}= & A_{y}\left(\gamma_{v}(n, m-1)\right) \cdot A_{x}\left(\varphi_{v}(n, m-1)\right) \\
& \cdot A_{z}\left(\theta_{v}(n, m-1)\right) \cdot y_{n, m-1}^{v}, \\
z_{n, m}^{v}= & A_{y}\left(\gamma_{v}(n, m-1)\right) \cdot A_{x}\left(\varphi_{v}(n, m-1)\right) \\
& \cdot A_{z}\left(\theta_{v}(n, m-1)\right) \cdot z_{n, m-1}^{v} .
\end{aligned}
$$

In (33) and (34), the matrix of rotation angle $\alpha$ around $y$-axis is

$$
A_{y}(\alpha)=\left\{\begin{array}{ccc}
\cos (\alpha) & 0 & \sin (\alpha) \\
0 & 1 & 0 \\
-\sin (\alpha) & 0 & \cos (\alpha)
\end{array}\right\}
$$

Therefore, the coupling transformation of the two moving coordinate systems at the node $P_{u v}$ can be obtained on the basis of formula (10) and (11). The coordinate of next node can be obtained based on the transformed moving coordinate system. All nodal coordinates on orthogonal curve net can be obtained by continuous iterative computation which contains node coordinate recursion and coupling transformation of moving coordinate system.

\section{Experimental Smart FBG Plate}

When the flexible plate structure deformation occurs, the surface would generate a strain. If the impact of environmental temperature changes is ignored, the strain detected by FBG

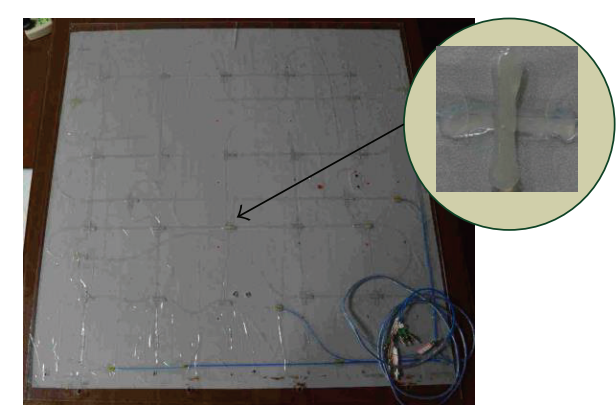

FIgURE 6: Schematic diagram of a smart FBG plate.

sensors bonded on the structure surface can be considered as strain of the flexible plate structure on the measuring point:

$$
\varepsilon=\frac{\Delta \lambda_{B}}{K_{\varepsilon}} .
$$

Here, $\varepsilon$ is the detected strain, $\Delta \lambda_{B}$ is the change of the fiber grating center wavelength, and $K_{\varepsilon}$ is the strain sensitivity coefficient. For particular fiber grating, $K_{\varepsilon}$ is constant.

While the FBG sensor is employed to detect the curvature in the plane of the plate surface,

$$
\rho=\frac{2}{h K_{\varepsilon}} \Delta \lambda_{B} .
$$

Here, $\rho$ is the curvature of measuring point and $h$ is the thickness of the plate. For a particular plate structure and FBG sensor, $h$ and $K_{\varepsilon}$ are constants.

The schematic diagram of a smart FBG plate is shown in Figure 6. A compensated FBG sensor is placed in the bottom of the plate to compensate the temperature influence of the FBG sensors.

Using the detected curvature, orthogonal curved network can be constructed by using continuous curvature. Orthogonal moving coordinate system on two directions for orthogonal curved network can be established. Node coordinates of orthogonal curvilinear net can be calculated using the moving coordinate system, and the internal relations of different nodes can be used to implement the coupling relationship between orthogonal coordinate systems. The iterative calculation of node coordinates and the transformation of moving coordinate system to realize all nodes' coordinate of orthogonal curved network constitute the two steps used to reconstruct the whole shape of curved surface.

To compare the vibration shape reconstruction performance, laser displacement sensor is employed in most researches. The diagram of the error analysis principle is shown in Figure 7.

As shown in Figure 7, the reconstruction accuracy validation process is as follows: low-frequency excitation of FBG is applied to structure by the exciter. The distributed FBG sensing network could get the changes of the wavelength of FBG grating measuring point. Then, on the basis of FBG wavelength and the structure strain relationship, these changes can be transferred into strain data. Then orthogonal curved network based reconstruction method can be 


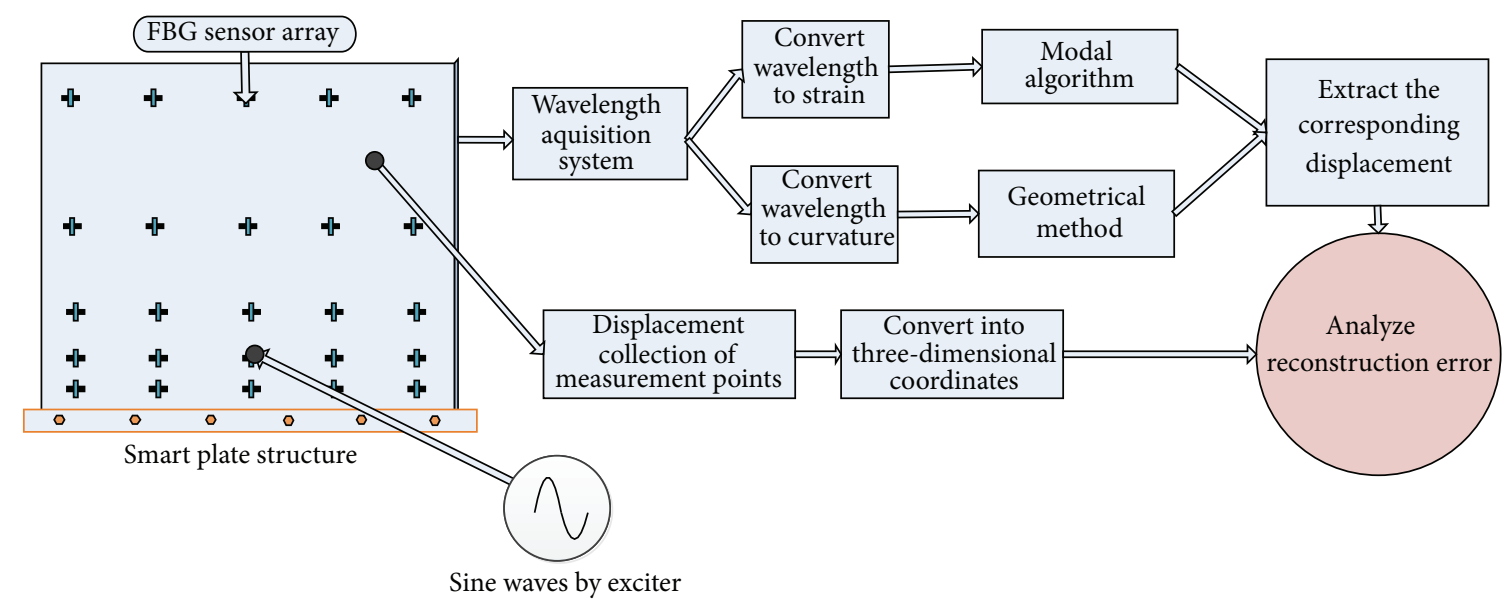

FIGURE 7: Error analysis principle for vibration shape reconstruction of smart FBG structure.

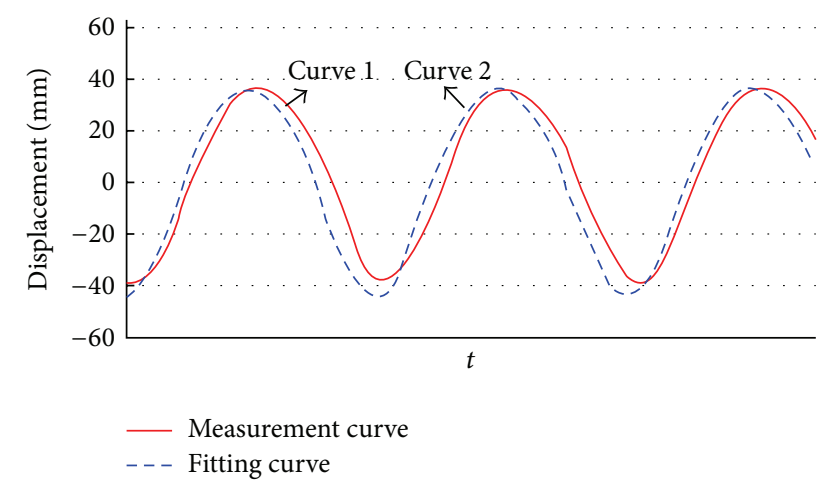

FIgURE 8: An example of the sampled dynamic data.

employed for the structure vibration shape reconstruction. In the same time, a laser displacement sensor is employed to measure the actual displacement of the structure testing point. Then the reconstruction error can be obtained by estimating the difference between the measured and estimated displacement.

\section{Dynamic Error Analysis Model}

As the essence of all these shape reconstruction algorithms is doing data transformation from the original strain data acquired from the distributed FBG sensors, the dynamic analysis method proposed in signal processing area can be employed. An example of the sampled dynamic data of geometrical reconstruction method can be shown in Figure 8. The solid line is the signal measured by the laser displacement sensor. The dashed line is the estimated signal by geometrical shape reconstruction algorithm. The lag of the two curves is the sampling delay of the two data acquisition systems. The strain data acquisition system is in advance of the laser displacement sensor data acquisition system.

Dynamic error analysis can be done by modelling of the data transmission process. The model can be treated as a combination of a FIR filter model with a noise. The diagram of the dynamic error analysis model is shown in Figure 9.

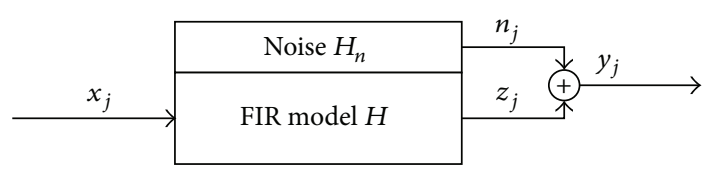

FIGURE 9: Diagram of the dynamic error analysis model.

For a specific measured point on the plate, $x_{j}$ is the actual displacement of the measured point in the plane of the plate surface. $z_{j}$ is the theoretical reconstructed or estimated displacement without reconstruction noise. $n_{j}$ is the reconstruction noise signal and $y_{j}$ is the sum of $z_{j}$ and $n_{j}$. The length of the FIR model $H$ is $M . z_{j}$ is the linear weighted sum of all past $M$ inputs:

$$
z_{j}=\sum_{i=1}^{M} w_{i} x_{i j} .
$$

Here, $w_{i}$ is the weighting coefficients, $x_{i j}$ is the input sequence, and $x_{1 j}=x_{j}, x_{2 j}=x_{j-1}, \ldots, x_{M j}=x_{j-M+1}$. Meanwhile, let $W=\left[w_{1}, w_{2}, \ldots, w_{M}\right]^{T}$ and $X_{j}=\left[x_{1 j}, x_{2 j}, \ldots\right.$, $\left.x_{M j}\right]$; then formula (15) can be written in vector form:

$$
z_{j}=X_{j} W
$$

LMS algorithm can be employed for model identification of the above dynamic error analysis model. The dynamic modelling principle is shown in Figure 10.

As shown in Figure 10, $y_{j}$ filtered by $F$ gets $s_{j}$. The output of the identification adaptive filter $H^{*}$ is $d_{j} . s_{j}$ minus $d_{j}$ is $e_{j}$. LMS 1 is used to adjust $W^{*}$ by minimizing the expectation $P$ of the mean square error of $e_{j}$. While the adaptive filter converges, $W^{*}$ is closer to the series of $H$ and $F$. Since $W_{f}$ is known, $\mathrm{H}_{2}{ }^{*}$ can be built. $x_{j}$ filtered through $\mathrm{H}_{2}{ }^{*}$ and $\mathrm{F}$ gets $s^{\prime}{ }_{j} . s^{\prime}{ }_{j}$ minus $d_{j}$ gets $e^{\prime}{ }_{j}$. LMS2 is used to adjust $W_{2}{ }^{*}$ by minimizing the expectation $P$ of the mean square error of $e^{\prime}{ }_{j}$. While the adaptive filter converges, $W_{2}{ }^{*}$ is almost the same with $W$. As the main purpose of LMS2 is to get the parameter 


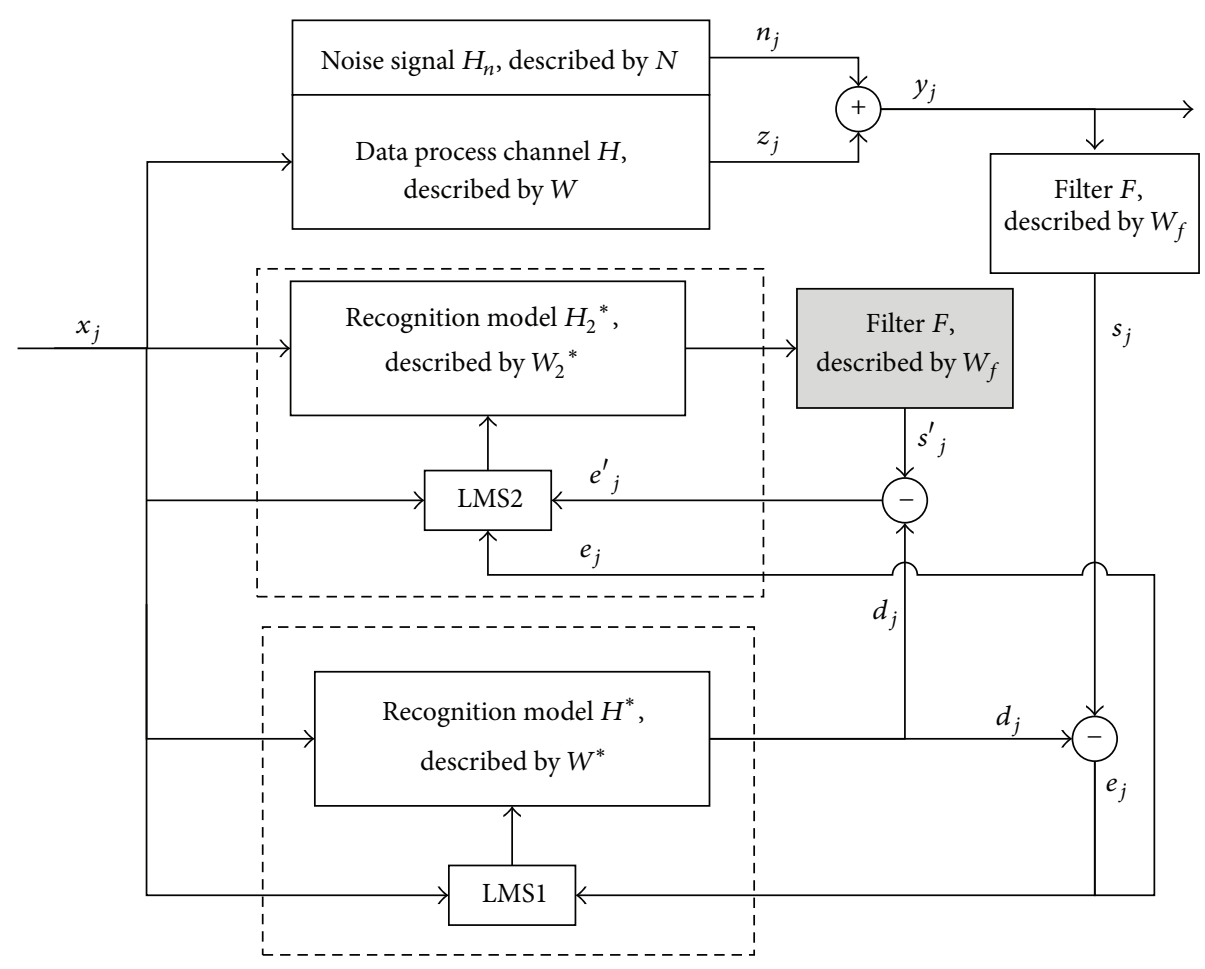

FIGURE 10: Dynamic modelling principle based on LMS algorithm.

of $H$, the exact parameter of $H^{*}$ is needed. $e_{j}$ can be used as the starting condition of LMS2 algorithm. Only when the expectation $P$ of the mean square error of $e_{j}$ is less than a certain value can LMS2 start.

With $W$, the amplitude attenuation, phase delay, and noise mixing can be calculated. In Figure 10,

$$
\begin{aligned}
y_{j} & =z_{j}+n_{j}, \\
e_{j} & =s_{j}-d_{j}, \\
e^{\prime}{ }_{j} & =s^{\prime}{ }_{j}-d_{j}, \\
s_{j} & =Y_{j} W_{f}, \\
s^{\prime} & =X_{j} W_{2}{ }^{*} W_{f}, \\
P & =E\left[e^{2}{ }_{j}\right], \\
P^{\prime} & =E\left[e^{\prime}{ }_{j}\right] .
\end{aligned}
$$

Using $\sigma$ to describe unit amplitude noise and assuming the amplitude of noise $N$ is $S$, the expectation $P_{N}=E\left[n_{j}\right]$;

$$
N=\sigma F_{j}+P_{N} .
$$

With the model parameter $\mathrm{W}_{2}{ }^{*}$ of $\mathrm{H}_{2}{ }^{*}$, the amplitude attenuation and phase delay can be obtained. The noise mixing can be obtained using (17) and (24). Therefore, the parameter identification of $W_{2}{ }^{*}$ is the key of reconstruction error analysis.

The nomenclature is shown in Nomenclature.

\section{Parameter Identification and Reconstruction Error Analysis}

To analyze the dynamic reconstruction error, identification of the model parameter $\mathrm{W}_{2}{ }^{*}$ of $\mathrm{H}_{2}{ }^{*}$ must be done.

5.1. Parameter Identification. The identification of $W_{2}{ }^{*}$ can be done using LMS algorithm. From (18) and (20), one can get

$$
\begin{aligned}
P= & E\left[\left(z_{f j}-z^{*}{ }_{j}\right)^{2}\right] \\
= & W^{* \tau} E\left[X_{j}{ }^{T} X_{j}\right] W^{*}-2 E\left[z_{f j} X_{j}\right] W^{*} \\
& +E\left[z_{f j}{ }^{2}\right] .
\end{aligned}
$$

Let

$$
\begin{aligned}
& Q=E\left[z_{f j} X_{j}\right], \\
& R=E\left[X_{j}{ }^{T} X_{j}\right], \\
& G=E\left[z_{f j}{ }^{2}\right] .
\end{aligned}
$$

Equation (28) can be written as

$$
P=W^{* T} R W^{*}-2 Q W^{*}+G .
$$

Equation (29) is a quadratic function of the weight vector $W^{*} . P \sim W^{*}$ is a concave hyperparaboloid with a unique minimum. Using the steepest descent method,

$$
W^{*}{ }_{j+1}=W^{*}{ }_{j}+2 u e_{j} X_{j} .
$$


Similarly,

$$
W_{2}{ }^{*}{ }_{j+1}=W_{2}{ }^{*}+2 u^{\prime} e^{\prime}{ }_{j} X_{j} .
$$

Here, $u, u^{\prime}$ are the adjusting step.

5.2. Reconstruction Error Analysis. Using the obtained model parameter $W_{2}{ }^{*}$, the reconstruction error including the amplitude attenuation, phase delay, and noise mixing can be analyzed.

The input signal frequency is $f$. The sampling period is $T$, $\tau=2 \pi f T$. The sinusoidal response is

$$
y_{j}=\sum_{i=1}^{N} w_{i} \sin [(j-i+1) \tau] .
$$

Expand (32):

$$
\begin{aligned}
y_{j}= & \sin (j \tau) \sum_{i=1}^{N} w_{i} \cos [(i-1) \tau] \\
& +\cos (j \tau) \sum_{i=1}^{N} w_{i} \sin [(1-i) \tau]
\end{aligned}
$$

Let

$$
\begin{aligned}
y_{j} & =\rho \sin (j \tau-\Delta \varphi), \\
A & =\sum_{i=1}^{N} w_{i} \cos [(i-1) \tau], \\
B & =\sum_{i=1}^{N} w_{i} \sin [(1-i) \tau] .
\end{aligned}
$$

Then

$$
\begin{aligned}
\psi & =\frac{A}{\cos [\operatorname{arctg}(B / A)]}, \\
\Delta \varphi & =-\operatorname{arctg} \frac{B}{A} .
\end{aligned}
$$

Here $\psi$ is the amplitude of the reconstruction data and $\Delta \varphi$ is the phase delay of reconstruction results respected to the actual vibration displacement. Equation (17) shows

$$
n_{j}=y_{j}-z_{j} .
$$

One can get

$$
n_{j}=y_{j}-X_{j} W .
$$

Using (40), $n_{j}$ can be calculated. The expectation and magnitude of the mixing signal can be obtained.

5.3. Design of Filter F. The mixing noise in the algorithm process could be filtered by filter $F$. As the mixing noise is high frequency, the filter $F$ should be a low pass filter. The upper limit frequency of the filter $F$ should be greater

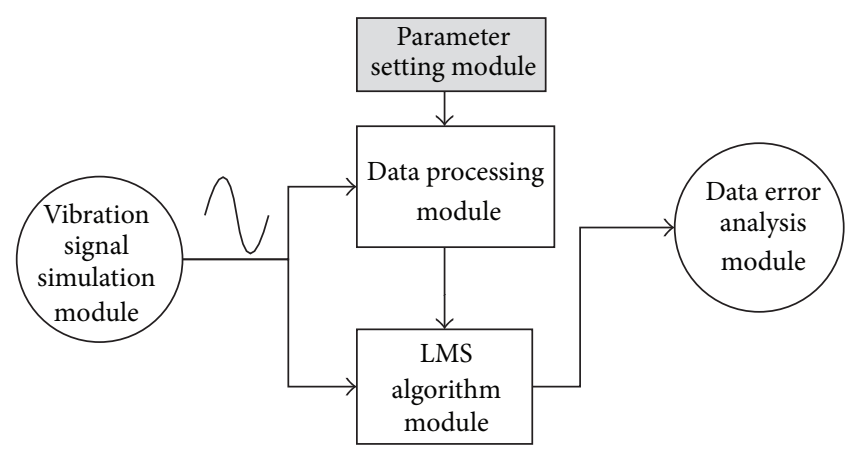

FIgURE 11: The diagram of software structure.

than main natural frequency of the structure. Filter $F$ can be designed with window function method. Assuming that the amplitude-frequency characteristic of this low-pass filter is $\left|H_{d}\left(e^{j \omega}\right)\right|=1$, the phase-frequency characteristic is $\varphi(\omega)=0$, and then the unit sample response of the filter is

$$
h_{d}(n)=\frac{1}{2 \pi} \int_{-\pi}^{\pi} H_{d}\left(e^{j \omega}\right) e^{j \omega n} d \omega=\frac{\sin \left(\omega_{c} n\right)}{\pi n} .
$$

Here $\omega_{c}$ is the upper limit of the low-frequency filter. As $h_{d}(n)$ is an infinite function which is symmetric around $h_{d}(0)$, it is a noncausal system. In order to make $h_{d}(n)$ physically realizable, $h_{d}(n)$ should be truncated with the length $M+$ $1: h_{d}(-M / 2), \ldots, h_{d}(0), \ldots, h_{d}(M / 2)$. Shifting the truncated $h_{d}(n)$, the following is obtained:

$$
h(n)=h_{d}\left(n-\frac{M}{2}\right), \quad n=0,1, \ldots, M .
$$

System (42) is causal system and has a similar frequency response with $h_{d}(n)$. While the phase frequency response of $H_{d}\left(e^{j \omega}\right)$ is $\varphi(\omega)=-M \omega / 2, \varphi(\omega)$ has linear phase. The lowpass filter can be obtained by (42):

$$
h_{d}(n)=\frac{\sin \left(\omega_{c}(n-M / n)\right)}{\pi(n-M / 2)} .
$$

Using (43) and the actual vibration frequency in the experimental procedure, low-pass filter $F$ can be constructed.

\section{Simulation and Experiment Platform}

6.1. Simulation Platform. The diagram of the simulation software is shown in Figure 11.

The vibration signal simulation module generates sinusoidal vibration signal. Data processing module is responsible for processing the amplitude, phase, and mixing noise of the data. The processing parameters are set by the parameter module. LMS algorithm module is responsible for dynamic model parameter identification. Data error analysis module is responsible for the analysis and calculation of the amplitude attenuation, phase delay, and noise mixing. The feasibility and effectiveness of dynamic error analysis method proposed in this paper could be verified by comparing the difference of the set parameter and the calculated error parameter. 


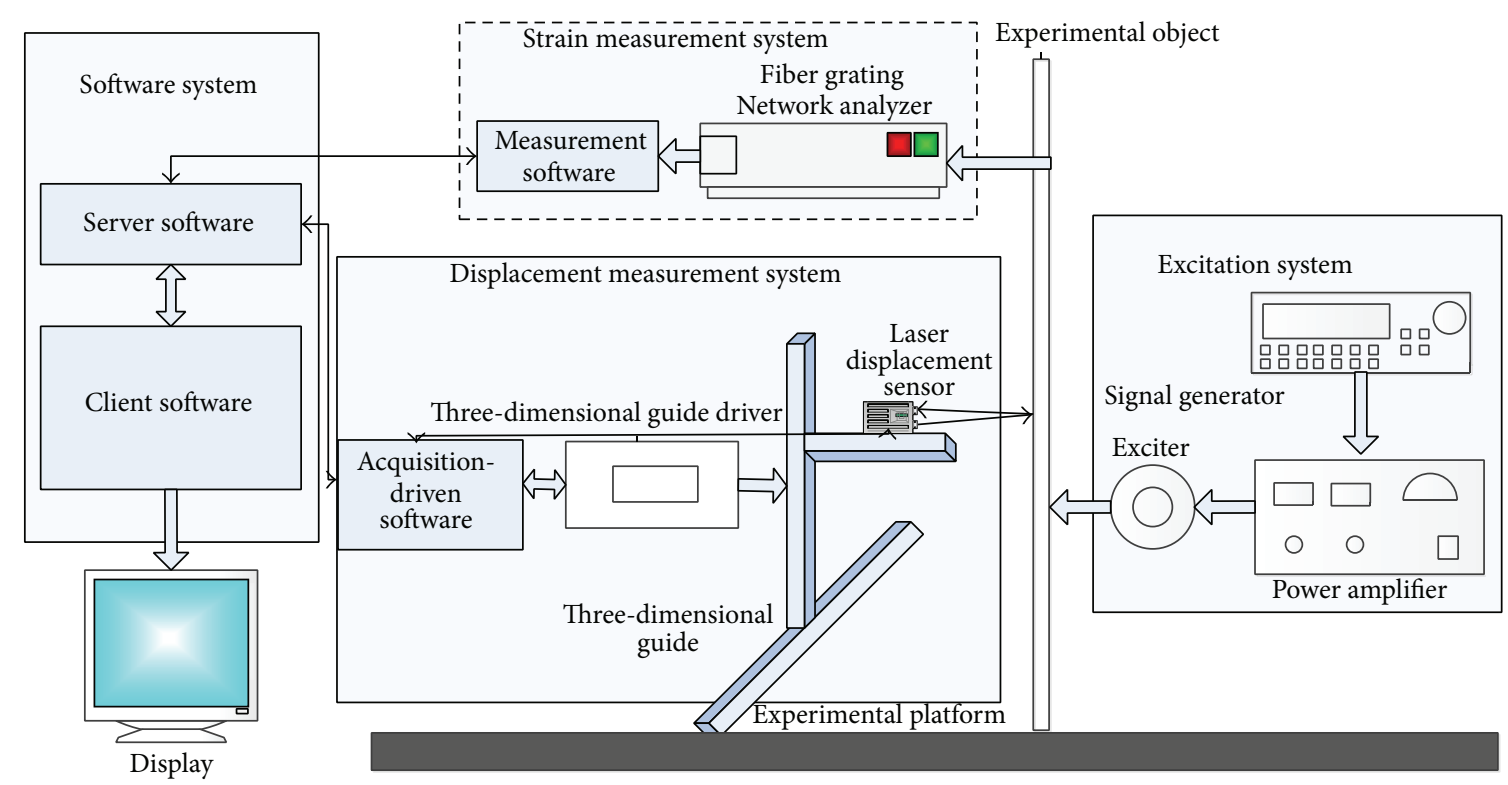

FIGURE 12: Schematic diagram of the experimental platform.

In order to improve the searching efficiency and accuracy, initial $u$ and $u^{\prime}$ are set to 0.01 . And the step reduces $10 \%$ in every iteration.

Define recognition accuracy as $\eta=(1-\mid$ set value identification value|/|set value|) $\cdot 100 \%$. A high recognition accuracy could verify a good dynamic error analysis capability.

6.2. Experiment Platform. A square PC plate is used to construct the smart plate. The elastic modulus is $6.9 \times 10^{9} \mathrm{~Pa}$, Poisson's ratio is 0.33 , the density is $1200 \mathrm{~kg} / \mathrm{m}^{3}$, and the size of the plate is $800 \times 800 \times 5 \mathrm{~mm}$. 25 orthogonal pairs of FBG sensors are bonded on the front and back of the plate. Each pair of the orthogonal FBG sensors can measure the structural strain in horizontal direction and vertical direction, respectively.

With the constructed smart FBG plate, a vibration shape reconstruction experiment platform is built as shown in Figure 12. The experimental platform contains a support base, the smart FBG plate, an excitation system, strain measurement system, displacement measurement system, and shape reconstruction software system. The support base of the experimental platform is an optical experiment table. The table type is GZ103PTB. The excitation system contains a signal generator, a power amplifier, and an exciter. The strain measurement system consisted of the FBG sensors and the fiber grating network analyzer. It can collect the grating wavelength signal of the FBG sensors precisely for strain and curvature calculation. Displacement measurement system consisted of three-dimensional guide, motion controller, motor driver, laser displacement sensor, and data acquisition software. It can achieve real-time acquisition of the vibration displacement of the smart FBG plate. The software system contains a server and a client. The server is used for data transmission of the original data. The client is used for real-time shape reconstruction. The reconstruction result can display dynamically and visually on the screen.

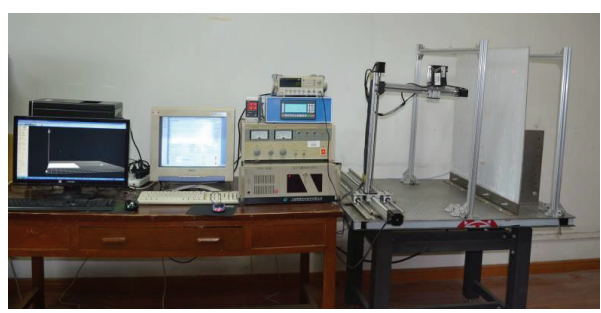

FIGURE 13: Photo of the experimental platform.

The photo of the experimental platform is shown in Figure 13. The range of FBG sensor central wavelength is $1532 \mathrm{~nm} \sim 1568 \mathrm{~nm}$. The type of the fiber grating network analyzer is FONA-2008C. Its precision is $1 \mathrm{pm}$. The type of the signal generator is SFG-2110. The type of the power amplifier is YE5872. The type of the exciter is JZK-10 with maximum exciting force of $200 \mathrm{~N}$. The model of laser displacement sensor is LK-GD500. The accuracy of the three-dimensional guide is $0.0025 \mathrm{~mm}$.

\section{Simulation and Experiment Results}

7.1. Shape Reconstruction Experiment. The proposed dynamic error analysis method can be used to value the shape reconstruction performance. To test the effectiveness of the proposed error analysis method, geometrical reconstruction method proposed in [19] is adopted in our simulation and experiment analysis. Sweeping frequency technique is used to find the natural frequency of the smart plate. While the vibration frequency is higher than the sixth natural frequency, the maximum vibration displacement of the smart plate is relatively small. So the shape reconstruction in our experiment is only done for the first six natural frequencies. The reconstructed vibration shape at the second natural frequency is shown in Figure 14. 
TABLE 1: Result of simulation analysis.

\begin{tabular}{|c|c|c|c|c|c|c|c|c|c|}
\hline Freq. & SA & DA & $\mathrm{ADP}$ & SDT & DDT & DDP & $\mathrm{SN}$ & $\mathrm{DN}$ & NDP \\
\hline $1.5 \mathrm{~Hz}$ & $96.00 \%$ & $95.84 \%$ & $99.83 \%$ & $0.10 T$ & $0.10 T$ & $100.00 \%$ & $0.50 \mathrm{~cm}$ & $0.57 \mathrm{~cm}$ & $86.00 \%$ \\
\hline $2.3 \mathrm{~Hz}$ & $85.00 \%$ & $85.05 \%$ & $99.94 \%$ & $0.20 T$ & $0.10 T$ & $100.00 \%$ & $0.40 \mathrm{~cm}$ & $0.46 \mathrm{~cm}$ & $85.00 \%$ \\
\hline $3.5 \mathrm{~Hz}$ & $99.00 \%$ & $99.45 \%$ & $99.55 \%$ & $0.35 T$ & $0.35 T$ & $100.00 \%$ & $0.60 \mathrm{~cm}$ & $0.62 \mathrm{~cm}$ & $96.67 \%$ \\
\hline $4.2 \mathrm{~Hz}$ & $90.00 \%$ & $90.73 \%$ & $99.19 \%$ & $0.30 T$ & $0.30 T$ & $100.00 \%$ & $0.80 \mathrm{~cm}$ & $0.92 \mathrm{~cm}$ & $85.00 \%$ \\
\hline $5.0 \mathrm{~Hz}$ & $80.00 \%$ & $80.16 \%$ & $99.80 \%$ & $0.05 T$ & $0.05 T$ & $100.00 \%$ & $0.30 \mathrm{~cm}$ & $0.34 \mathrm{~cm}$ & $86.67 \%$ \\
\hline $5.9 \mathrm{~Hz}$ & $98.00 \%$ & $98.83 \%$ & $99.15 \%$ & $0.15 T$ & $0.15 T$ & $100.00 \%$ & $0.20 \mathrm{~cm}$ & $0.23 \mathrm{~cm}$ & $85.00 \%$ \\
\hline
\end{tabular}

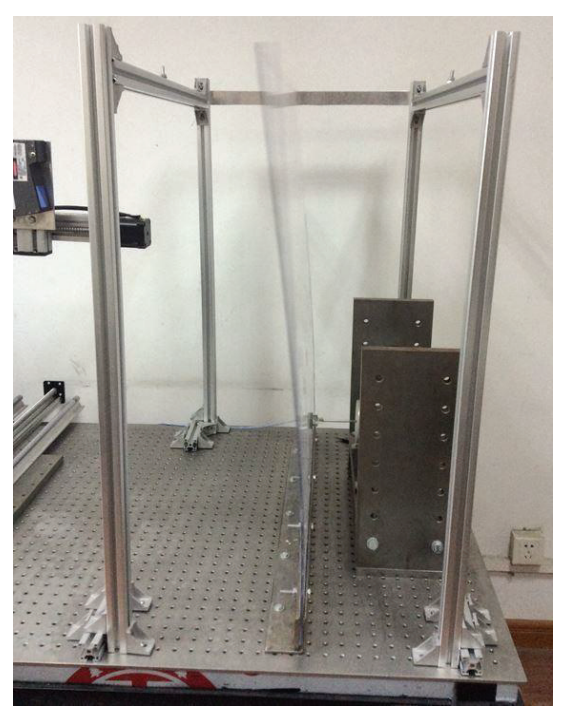

(a) The actual vibration shape

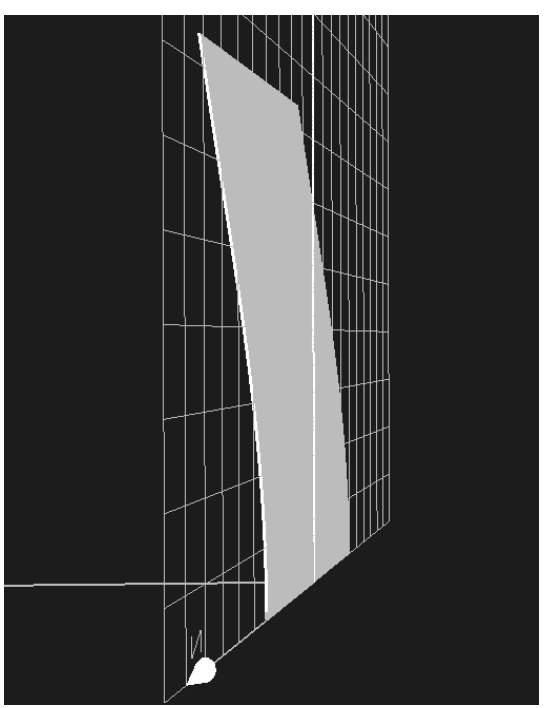

(b) The reconstructed shape

FIGURE 14: Shape reconstruction for the second modal.

7.2. Simulation Verification. To test the effectiveness of proposed dynamic error analysis algorithm, six different excitations are applied. $2.3 \mathrm{~Hz}, 4.2 \mathrm{~Hz}$, and $5.9 \mathrm{~Hz}$ are the first three resonance frequencies and $1.5 \mathrm{~Hz}, 3.5 \mathrm{~Hz}$, and $5.0 \mathrm{~Hz}$ are nonresonance frequencies. The simulation results are shown in Table 1.

In Table 1, SA represents set attenuation of the output signal. DA represents distinguished attenuation with the error analysis system. ADP represents attenuation distinguished precision of the error analysis method. SDT represents set delay time of the signal phase. DDT represents attenuation delay time of the signal phase. DDP represents delay distinguished precision of the signal phase with the error analysis method. SN represents set noise of the actual output signal. $\mathrm{DN}$ represents distinguished noise with the error analysis system. NDP represents noise distinguished precision of the error analysis method. The frequencies of $2.3 \mathrm{~Hz}, 4.2 \mathrm{~Hz}$, and $5.9 \mathrm{~Hz}$ are the first three modal frequencies of FBG smart plate structure. As shown in Table 1, for different vibration frequencies, the identification accuracy of signal attenuation value is not less than $99.15 \%$. The identification accuracy of the signal delay is $100.00 \%$; the identification accuracy of the noise mixing is not less than $85.00 \%$. It can be seen that the algorithm can be set to accurately identify the errors of different amplitude attenuation, phase delay, and noise mixing. The identification accuracy of the amplitude attenuation and phase delay is higher, and noise identification is lower. Figure 15 shows the convergence process of the proposed algorithm. Figure 15(a) is the convergence process of LMS1 algorithm, and Figure 15(b) is the convergence process of LMS2 algorithm. It is shown that the proposed algorithm could rapidly converge in the calculation process, indicating that the algorithm has a good searching capability.

7.3. Experimental Verification. In the vibration experiments, the excitation frequencies are the same as in Table $1.2 .3 \mathrm{~Hz}$, $4.2 \mathrm{~Hz}$, and $5.9 \mathrm{~Hz}$ are the first three resonance frequencies and $1.5 \mathrm{~Hz}, 3.5 \mathrm{~Hz}$, and $5.0 \mathrm{~Hz}$ are nonresonance frequencies. The reconstruction experiment results for the first three modals are shown in Figure 16. The performance of the proposed reconstruction algorithm is very good. The fitting curve and the actual curve are nearly the same.

Using traditional static error analysis method, the calculation equation for reconstruction accuracy is shown as follows:

$$
\eta_{1}=\frac{\sum_{t=1}^{T}\left(1-|E(t)| / E^{\prime}(t)\right)}{T} \cdot 100 \%
$$

Here, $T$ is the number of the measurement points and $E(t)$ is the difference between the measuring displacement and reconstruction displacement for a measurement point 


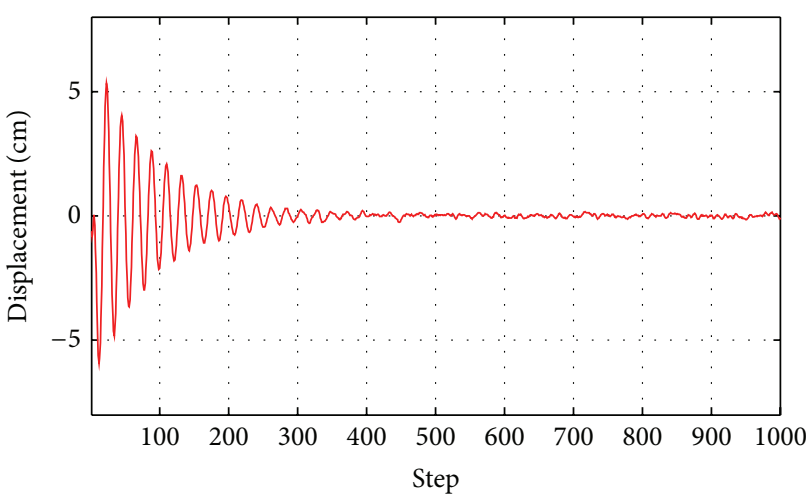

(a) Convergence of $e_{j}$

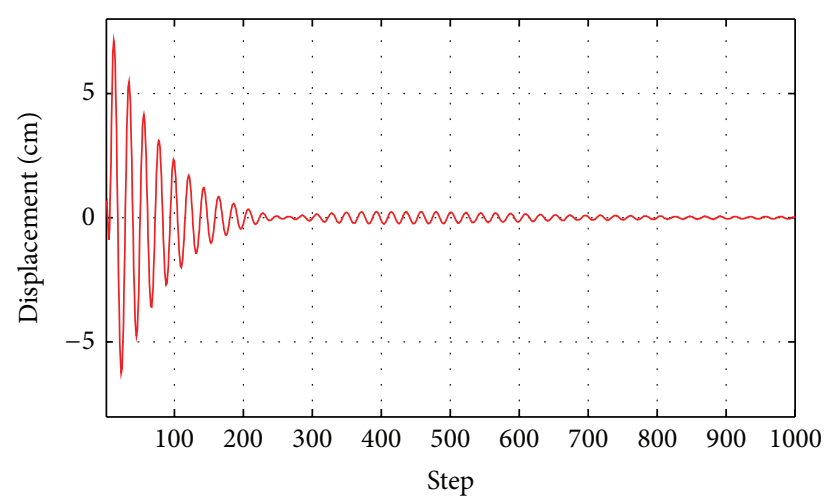

(b) Convergence of $e^{\prime}{ }_{j}$

FIGURE 15: LMS algorithm convergence process.

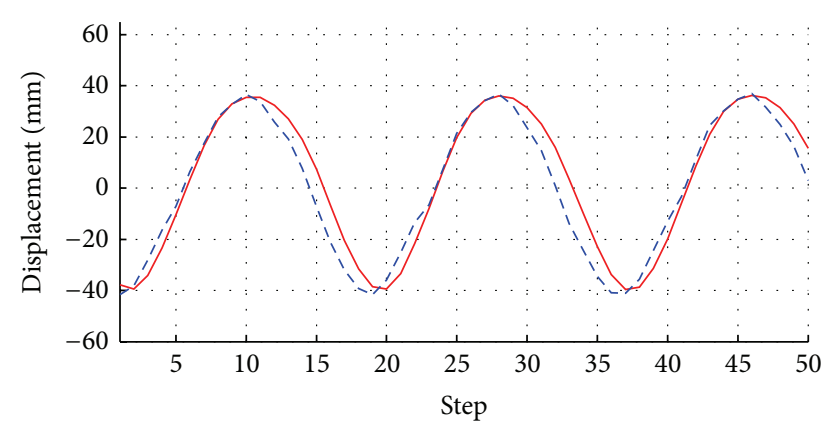

- Measurement curve
- - Fitting curve

(a) Experimental result at $2.3 \mathrm{~Hz}$

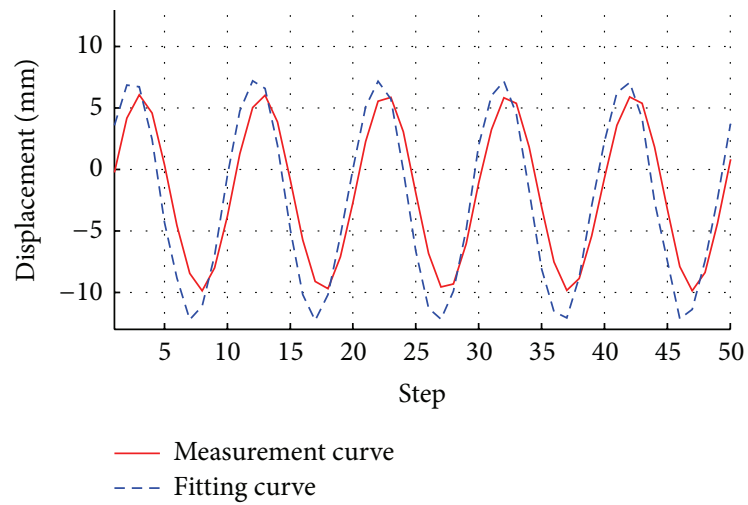

(b) Experimental result at $4.2 \mathrm{~Hz}$

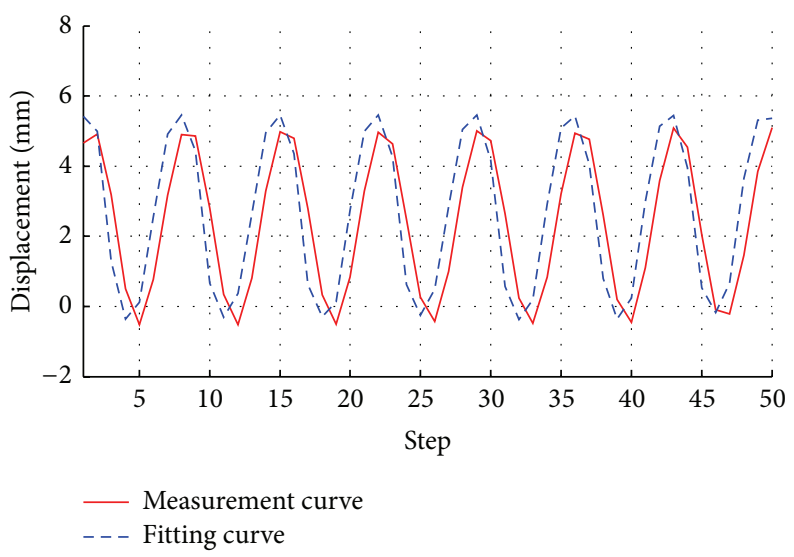

(c) Experimental result at $5.9 \mathrm{~Hz}$

FIGURE 16: The comparison between actual vibration waveform and reconstruction waveform.

at the time $t . E^{\prime}(t)$ is the measuring displacement of the measurement point measured at the time $t$.

Our proposed method can measure the ratio $\rho$ between the actual vibration amplitude and reconstructed vibration amplitude. The calculation equation of the reconstruction accuracy is

$$
\eta_{2}=(1-|1-\rho|) \cdot 100 \% .
$$

As shown in Table $1, \eta_{1}$ and $\eta_{2}$ are similar, indicating that our proposed algorithm could also value the static reconstruction performance. But amplitude only gives limited information for the reconstruction performance.

Using the obtained actual displacement data and the reconstruction displacement data, the dynamic error analysis results are shown in Table 2.

As shown in Table 2, the phase delay of each experiment varies in the range $16.67 \mathrm{~ms} \sim 20.30 \mathrm{~ms}$, indicating that the phase delay is a fixed delay time value. The delay time is much less than the minimum vibration period, $169.5 \mathrm{~ms}$ (at $5.9 \mathrm{~Hz}$ ), indicating that the reconstruction algorithm has 
TABLE 2: Data error analysis of the reconstruction result.

\begin{tabular}{lccccc}
\hline $\begin{array}{l}\text { Vibration } \\
\text { frequency }\end{array}$ & $\eta_{1}$ & $\eta_{2}$ & Phase delay & Mixing noise amplitude & Mixing noise expectation \\
\hline $1.5 \mathrm{~Hz}$ & $93.41 \%$ & $96.37 \%$ & $20.05 \mathrm{~ms}$ & $0.54 \mathrm{~cm}$ & $-0.18 \mathrm{~cm}$ \\
$2.3 \mathrm{~Hz}$ & $87.05 \%$ & $89.05 \%$ & $21.07 \mathrm{~ms}$ & $0.40 \mathrm{~cm}$ & $-0.10 \mathrm{~cm}$ \\
$3.5 \mathrm{~Hz}$ & $79.94 \%$ & $79.95 \%$ & $17.11 \mathrm{~ms}$ & $0.28 \mathrm{~cm}$ & $-0.06 \mathrm{~cm}$ \\
$4.2 \mathrm{~Hz}$ & $83.14 \%$ & $85.49 \%$ & $16.67 \mathrm{~ms}$ & $0.19 \mathrm{~cm}$ & $-0.02 \mathrm{~cm}$ \\
$5.0 \mathrm{~Hz}$ & $78.64 \%$ & $75.80 \%$ & $18.04 \mathrm{~ms}$ & $0.14 \mathrm{~cm}$ & $0.00 \mathrm{~cm}$ \\
$5.9 \mathrm{~Hz}$ & $73.53 \%$ & $72.14 \%$ & $20.30 \mathrm{~ms}$ & $0.09 \mathrm{~cm}$ & $0.03 \mathrm{~cm}$ \\
\hline
\end{tabular}

a good following ability. The algorithm can also identify the amplitude and expectation of the mixing noise. As the vibration frequency increases, the vibration amplitude decreases. Meanwhile the mixing noise introduced by the algorithm is also decreased.

\section{Conclusion}

A new dynamic data error analysis method is proposed in this paper. The detailed analysis process is illustrated, including the construction of the dynamic error analysis model and the LMS algorithm based parameter identification method. Simulation and experiment results show that the proposed dynamic error analysis method can accurately obtain the dynamic characteristics of the FBG smart plate structure reconstruction algorithm including signal amplitude attenuation, phase delay, and noise mixing. It can provide a theoretical basis and technical support for the improvement and engineering realization of various shape reconstruction algorithms. It can also apply to some other applications that need dynamic data analysis.

\section{Nomenclature}

$x_{j}$ : Vibration signal at time $j$ of a testing point on the structure surface

$y_{j}$ : Reconstructed output

$H$ : System model

$z_{j}$ : Theoretical output

$n_{j}$ : The introduced error

F: $\quad$ Filter

$s_{j}: \quad$ Filtered $y_{j}$

$H^{*}$ : The adaptive filter to identify $H+F$

$d_{j}$ : Output of $H^{*}$

$e_{j}$ : Difference of $s_{j}$ and $d_{j}$

$W^{*}$ : The identified model of $H+F$

$\mathrm{H}_{2}{ }^{*}$ : The estimated model of $\mathrm{H}$

$s^{\prime}{ }_{j}: \quad$ Filter output

$e^{\prime}{ }_{j}$ : Difference of $s^{\prime}{ }_{j}$ and $d_{j}$

$W_{2}{ }^{*}$ : Model for $H$.

\section{Conflict of Interests}

The authors declare that there is no conflict of interests regarding the publication of this paper.

\section{Acknowledgments}

This paper is sponsored by program of National Natural Science Foundation of China (no. 51175319), Innovation program of Shanghai Municipal Education Commission (no. 13ZZ075), and Shanghai Key Laboratory of Power Station Automation Technology.

\section{References}

[1] U. Baneen and J. E. Guivant, "A 2D Bayesian approach for damage detection in plate-type structures," Insight-NonDestructive Testing and Condition Monitoring, vol. 57, no. 3, pp. 144-152, 2015.

[2] Y. Zhong, S. Yuan, and L. Qiu, "Multi-impact source localisation on aircraft composite structure using uniform linear PZT sensors array," Structure and Infrastructure Engineering, vol. 11, no. 3, pp. 310-320, 2015.

[3] D. P. Thambiratnam, H. H. Shih, and T. H. Chan, "Structural health monitoring-use of mathematics and computers," in Proceedings of the International Conference on Mathematics and Computers in Sciences and in Industry (MCSI '14), pp. 55-59, Varna, Bulgaria, September 2014.

[4] J. Weinzettel, M. Reenaas, C. Solli, and E. G. Hertwich, "Life cycle assessment of a floating offshore wind turbine," Renewable Energy, vol. 34, no. 3, pp. 742-747, 2009.

[5] P. Li, Y. Liu, and J. Leng, "A new deformation monitoring method for a flexible variable camber wing based on fiber Bragg grating sensors," Journal of Intelligent Material Systems and Structures, vol. 25, no. 13, pp. 1644-1653, 2014.

[6] C. Wang and L. Cheng, "Use of fiber Bragg grating sensors for monitoring concrete structures with prestressed near-surface mounted carbon fiber-reinforced polymer strips," Journal of Intelligent Material Systems and Structures, vol. 25, no. 2, pp. 164-173, 2014.

[7] Y. Shapiro, G. Kosa, and A. Wolf, "Shape tracking of planar hyper-flexible beams via embedded PVDF deflection sensors," IEEE/ASME Transactions on Mechatronics, vol. 19, no. 4, pp. 1260-1267, 2014.

[8] T. Liu, A. W. Burner, T. W. Jones, and D. A. Barrows, "Photogrammetric techniques for aerospace applications," Progress in Aerospace Sciences, vol. 54, pp. 1-58, 2012.

[9] Z.-C. Qiu, X.-T. Zhang, X.-M. Zhang, and J.-D. Han, "A visionbased vibration sensing and active control for a piezoelectric flexible cantilever plate," Journal of Vibration and Control, 2014.

[10] C. T. Kiang, A. Spowage, and C. K. Yoong, "Review of control and sensor system of flexible manipulator," Journal of Intelligent 
\& Robotic Systems: Theory and Applications, vol. 77, no. 1, pp. 187-213, 2015.

[11] S. W. Park, H. S. Park, J. H. Kim, and H. Adeli, “3D displacement measurement model for health monitoring of structures using a motion capture system," Measurement, vol. 59, pp. 352-362, 2015.

[12] H.-I. Kim, L.-H. Kang, and J.-H. Han, "Shape estimation with distributed fiber Bragg grating sensors for rotating structures," Smart Materials and Structures, vol. 20, no. 3, Article ID 035011, 2011.

[13] L. Li, W. Li, P. Ding, X. Zhu, and W. Sun, "Structural shape reconstruction through modal approach using strain gages," in Computational Intelligence, Networked Systems and Their Applications, vol. 462 of Communications in Computer and Information Science, pp. 273-281, Springer, Berlin, Germany, 2014.

[14] H.-J. Bang, H.-I. Kim, and K.-S. Lee, "Measurement of strain and bending deflection of a wind turbine tower using arrayed FBG sensors," International Journal of Precision Engineering and Manufacturing, vol. 13, no. 12, pp. 2121-2126, 2012.

[15] A. Derkevorkian, S. F. Masri, J. Alvarenga, H. Boussalis, J. Bakalyar, and W. L. Richards, "Strain-based deformation shapeestimation algorithm for control and monitoring applications," AIAA Journal, vol. 51, no. 9, pp. 2231-2240, 2013.

[16] H.-J. Bang, S.-W. Ko, M.-S. Jang, and H.-I. Kim, "Shape estimation and health monitoring of wind turbine tower using a FBG sensor array," in Proceedings of the IEEE International Instrumentation and Measurement Technology Conference (I2MTC '12), pp. 496-500, IEEE, Graz, Austria, May 2012.

[17] Z.-C. Wang, D. Geng, W.-X. Ren, and H.-T. Liu, "Strain modes based dynamic displacement estimation of beam structures with strain sensors," Smart Materials and Structures, vol. 23, no. 12, Article ID 125045, 2014.

[18] H. Xu, W. X. Ren, and Z. C. Wang, "Deflection estimation of bending beam structures using fiber bragg grating strain sensors," Advances in Structural Engineering, vol. 18, no. 3, pp. 395-404, 2015.

[19] J. Yi, X. Zhu, H. Zhang, L. Shen, and X. Qiao, "Spatial shape reconstruction using orthogonal fiber Bragg grating sensor array," Mechatronics, vol. 22, no. 6, pp. 679-687, 2012.

[20] X. Qiao, H. Zhang, J. Xu, and X. Zhu, "Research and simulation of surface fitting algorithm based on surface patches splicing," in AsiaSim 2012, Communications in Computer and Information Science, pp. 286-295, Springer, Berlin, Germany, 2012.

[21] K.-S. Choi, Y.-H. Huh, I.-B. Kwon, and D.-J. Yoon, "A tip deflection calculation method for a wind turbine blade using temperature compensated FBG sensors," Smart Materials and Structures, vol. 21, no. 2, Article ID 025008, 2012.

[22] M. Li, H. Zhang, K. Liu, and X. Zhu, "Experimental platform design and implementation for plate structure shape perception and reconstruction algorithm," in Computational Intelligence, Networked Systems and Their Applications, vol. 462 of Communications in Computer and Information Science, pp. 199-209, Springer, Berlin, Germany, 2014.

[23] S. Rapp, L.-H. Kang, J.-H. Han, U. C. Mueller, and H. Baier, "Displacement field estimation for a two-dimensional structure using fiber Bragg grating sensors," Smart Materials and Structures, vol. 18, no. 2, Article ID 025006, 2009.

[24] H.-I. Kim, J.-H. Han, and H.-J. Bang, "Real-time deformed shape estimation of a wind turbine blade using distributed fiber Bragg grating sensors," Wind Energy, vol. 17, no. 9, pp. 1455-1467, 2014.
[25] J. Yi, H. Zhang, X. Qiao, and X. Zhu, "Shape monitoring for wing structure using fiber Bragg grating sensors," in Proceedings of the IEEE 5th International Conference on Advanced Computational Intelligence (ICACI '12), pp. 1032-1036, Nanjing, China, October 2012.

[26] J. Xu, H. Zhang, X. Zhu, L. Li, and P. Ding, "Curve surface fitting based on an improved genetic algorithm," in Proceedings of the 6 th International Congress on Image and Signal Processing (CISP '13), pp. 747-752, IEEE, Hangzhou, China, December 2013.

[27] S. Sengupta and S. K. Ghorai, "An efficient method for determining transmission characteristics of superstructure fiber Bragg grating and its use for multiparameter sensing," Optical Fiber Technology, vol. 21, pp. 146-153, 2015.

[28] M. S. Islam and A. B. Mohammad, "Transmission characteristics of Mach-Zehnder interferometer comb filter based on thermal operation," Applied Physics B: Lasers and Optics, vol. 117, no. 1, pp. 59-65, 2014.

[29] S. Mohapatra, A. Kar, and M. Chandra, "Advanced adaptive mechanisms for active noise control: a technical comparison," in Proceedings of the International Conference on Medical Imaging, $m$-Health and Emerging Communication Systems (MedCom '14), pp. 370-375, IEEE, Greater Noida, India, November 2014.

[30] M. Xiang and T. Wei, "Autobalancing of high-speed rotors suspended by magnetic bearings using LMS adaptive feedforward compensation," Journal of Vibration and Control, vol. 20, no. 9, pp. 1428-1436, 2014.

[31] S. Roopa and S. V. Narasimhan, “Transform domain Variable Step-size Griffiths Least Mean Square adaptive algorithm and its applications," Computers \& Electrical Engineering, vol. 40, no. 4, pp. 1028-1041, 2014.

[32] M. S. Salman, "Sparse leaky-LMS algorithm for system identification and its convergence analysis," International Journal of Adaptive Control and Signal Processing, vol. 28, no. 10, pp. 10651072, 2014.

[33] G. Su, J. Jin, Y. Gu, and J. Wang, "Performance analysis of $l_{0}$ norm constraint least mean square algorithm," IEEE Transactions on Signal Processing, vol. 60, no. 5, pp. 2223-2235, 2012.

[34] M. A. Z. Raja and N. I. Chaudhary, "Two-stage fractional least mean square identification algorithm for parameter estimation of CARMA systems," Signal Processing, vol. 107, pp. 327-339, 2015. 


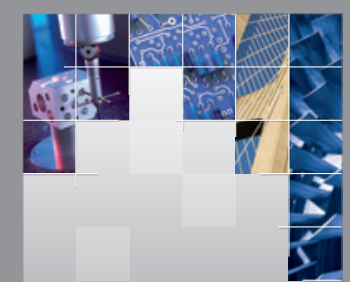

\section{Enfincering}
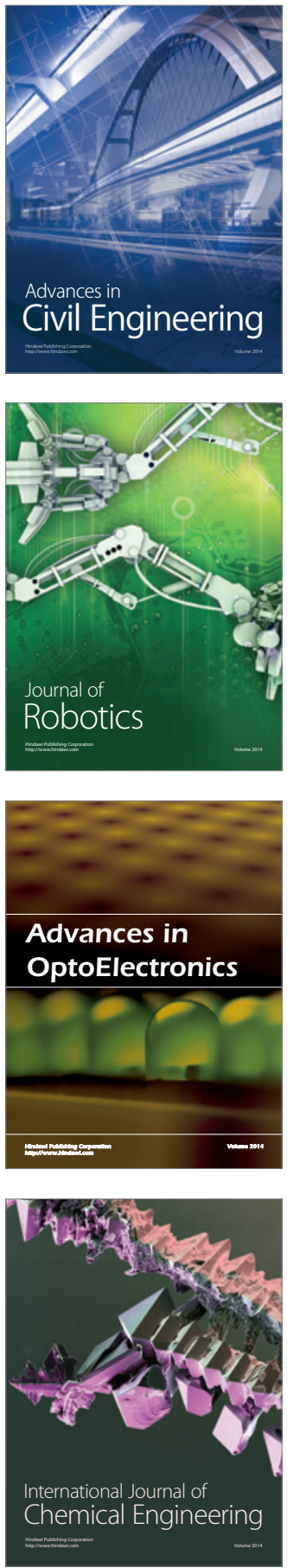

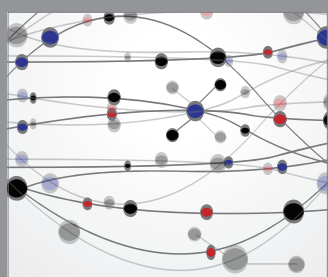

The Scientific World Journal

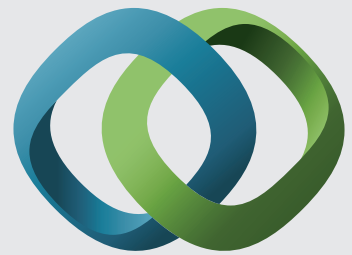

\section{Hindawi}

Submit your manuscripts at

http://www.hindawi.com
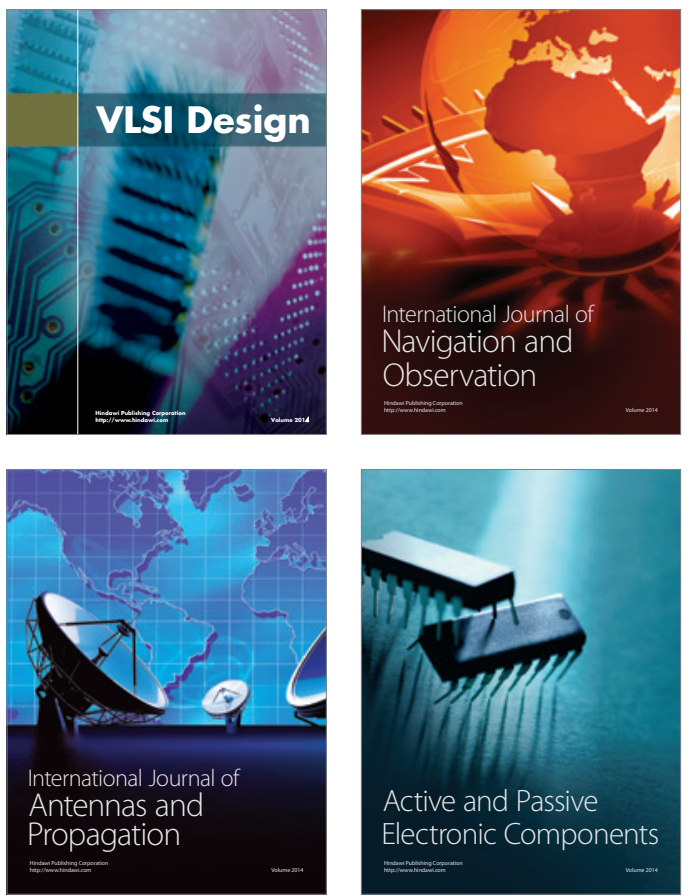
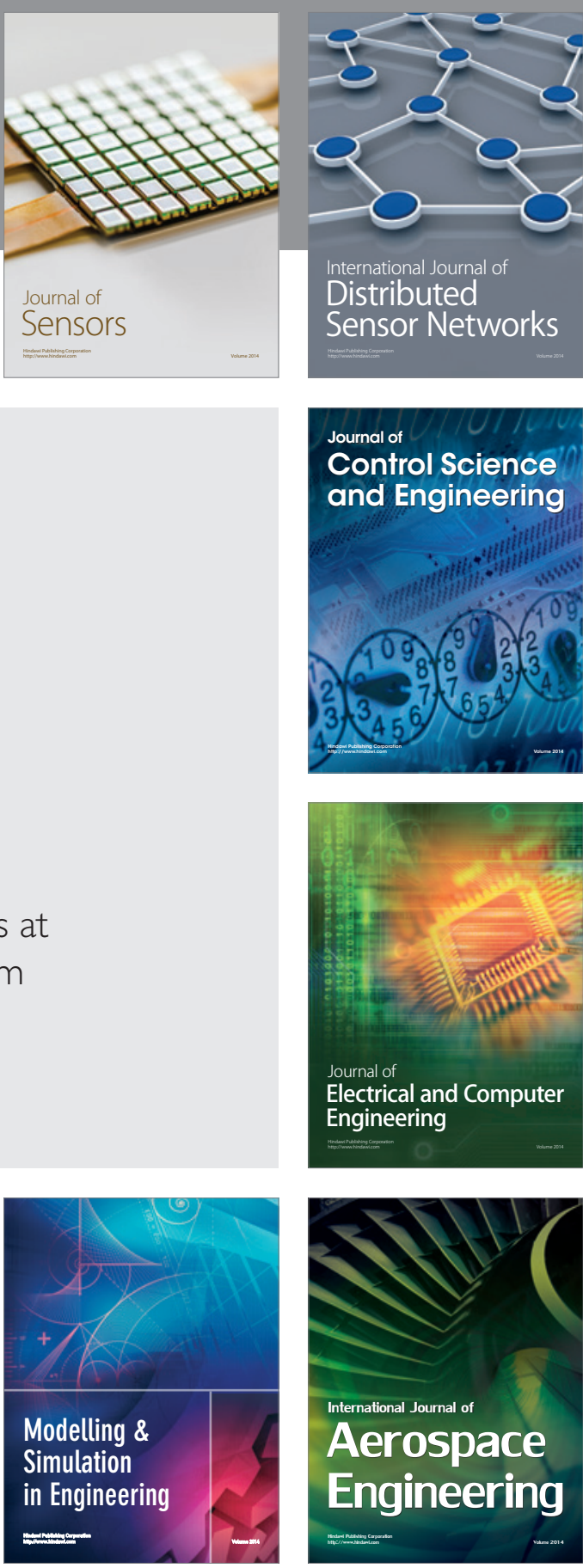

International Journal of

Distributed

Sensor Networks

Journal of

Control Science

and Engineering
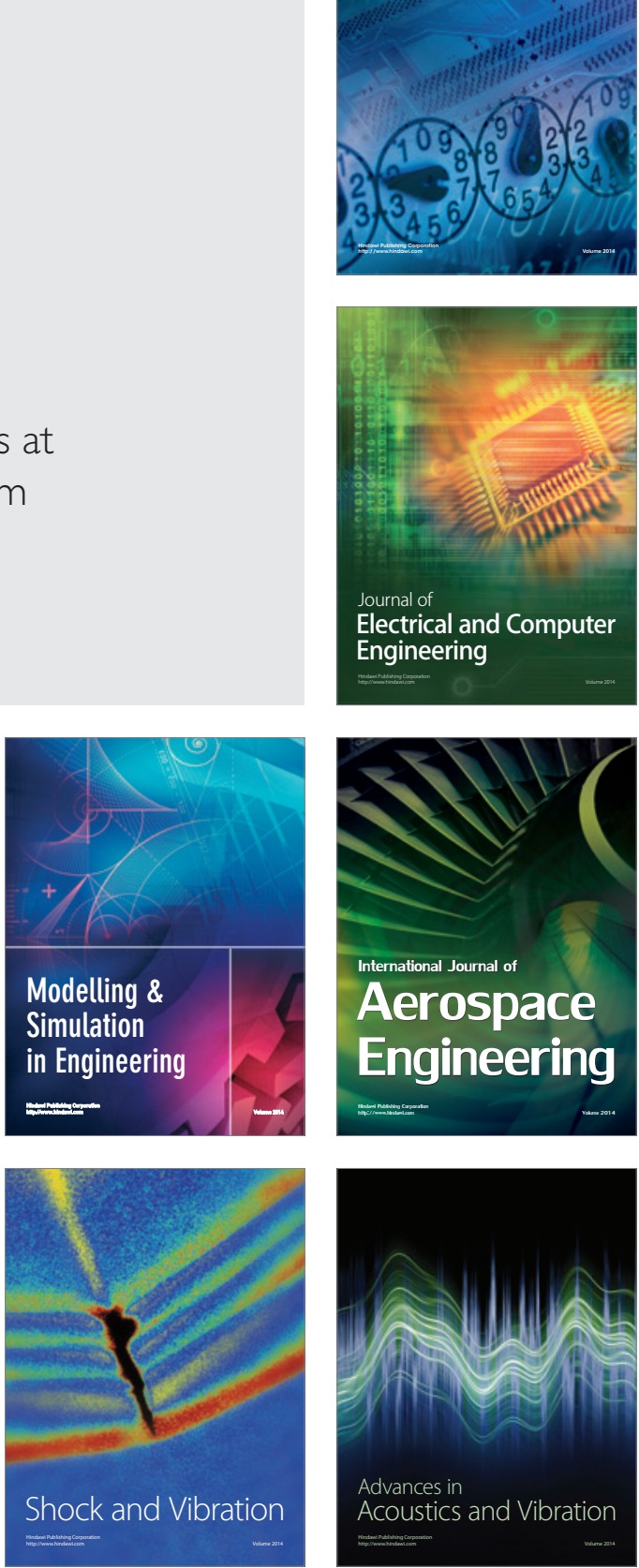